\title{
Impact of job burnout on mental health among social workers in public and private sector in Greece
}

\author{
Konstantinos Tsaras ${ }^{1}$, Evangelia Christopoulou ${ }^{2}$, Maria Malliarou ${ }^{1}$, Evangelos C. Fradelos ${ }^{3}$, \\ Stiliani Kotrotsiou ${ }^{1}$, Ioanna V. Papathanasiou ${ }^{1}$. \\ ${ }^{1}$ General Department, University of Thessaly, Larisa, GREECE. \\ ${ }^{2}$ Direction of Social Welfare, Municipality of Thessaly, Larisa, GREECE. \\ ${ }^{3}$ Psychiatric Department, Athens General Hospital for Thoracic Diseases "Sotiria", Athens, \\ GREECE
}

\begin{abstract}
.
Purpose: This study examines the impact of job burnout on the social workers' mental health in public and private sector.

Material and Method: A descriptive, cross-sectional study was conducted in which 103 social workers who were working in public $(n=56)$ and private $(n=47)$ sector of the Thessaly region in Greece. Data were collected with a questionnaire including socio-demographic and work-related characteristics, the Maslach Burnout Inventory - Human Services Survey (MBI-HSS) and the General Health Questionnaire (GHQ-28). Independent t-test, anova and Pearson coefficient were used in statistical analysis.

Results: Emotional exhaustion was positively related to somatic symptoms $(r=0.470, p<0.001)$, anxiety/insomnia $(r=0.429, \mathrm{p}<0.001)$, social dysfunction $(r=0.365, \mathrm{p}<0.001)$, depression $(\mathrm{r}=0.252$, $\mathrm{p}=0.010)$ and overall mental burden $(\mathrm{r}=0.518, \mathrm{p}<0.001)$ of social workers. Personal achievements were negatively related to somatic symptoms $(r=-0.326, p=0.001)$, anxiety/insomnia $(r=-0.266$, $\mathrm{p}=0.007)$, social dysfunction $(\mathrm{r}=-0.321, \mathrm{p}=0.001)$, depression $(\mathrm{r}=-0.444, \mathrm{p}<0.001)$ and overall mental burden $(\mathrm{r}=-0.444, \mathrm{p}<0.001)$. Also, depersonalization was positively associated with somatic symptoms $(r=0.218, p=0.027)$, anxiety/insomnia $(r=0.317, p=0.001)$ and overall mental burden $(\mathrm{r}=0.258, \mathrm{p}=0.009)$.
\end{abstract}

Conclusion: All dimensions of burnout had a significant effect on mental health disorders of social workers in labor.

Keywords: Burnout, satisfaction, mental health, depression, stress, social workers 